\title{
Implementation of Energy Efficient Routing Protocol for WSNs
}

\author{
Gaurav Dubey \\ M.Tech (IT) \\ IET, Alwar \\ Rajasthan, India
}

\author{
Alankar Shastri \\ Research Scholar \\ ITM, Gwalior \\ MP, India
}

\author{
Mohit Khandelwal \\ Research Scholar \\ IET Alwar \\ Rajasthan, India
}

\begin{abstract}
The wireless sensor network technology is an enter constituent for universally acclaimed communication. A wireless sensor network consists of a huge number of sensor nodes. Each sensor node senses environmental circumstances such as temperature, light, force and sends the sensed data to a base station which is an extensive way rancid in universe. Since the sensor nodes are powered by inadequate power batteries, is regulate to lengthen the life time of the network, low energy expenditure is important for sensor nodes. A technique is proposed for optimization of power consumption in wireless sensor network. Here some energy efficient protocols are present, developed from conventional clustering protocol of Federal and distributed clustering protocol. The simulations are performed on omnet++ and which prove that the proposed protocol is better than LEACH and other approaches available.
\end{abstract}

\section{Keywords}

Clustering, wireless sensor network. FSCP, Federal Approach, distributed Approach, FND, HND.

\section{INTRODUCTION}

It has established that, the best location for a sink is the center of circular deployment area in terms of energy efficiency. This approach of deployment is widely accepted in applications of large scale wireless sensor networks, such as environmental monitoring. However, as an intrinsic property of such deployment, brings the disturbed spatial distribution of energy consumption of sensor nodes. The enlarge in energy consumption around the sink would neutralize the low power plan for sensor nodes, and significantly decrease the network lifetime in sight of disconnected sink. Furthermore, this occurrence becomes prominent as the monitored area enlarges. Large-scale consumption of wireless sensor network takes up a lot of time and resources. Due to the important deterioration in network lifetime, whether the reasonable performance of WSN meets its design requirements for data collection is uncertain. Consequently, in terms of a precise solution of sensor nodes, an investigative technique on the network energy consumption is wanted in pre-deployment analysis and examination. We present some energy efficient protocols, developed from conventional clustering protocol of Federal and distributed clustering protocol. We perform simulation omnet++ and prove that our proposed protocol is better than LEACH another approach.

\section{RELATED WORK}

Jin-Shyan Lee in at al [1] proposed overcome the challenge of using fuzzy logic on the base station to determine the optimal number of CHs. Once this value is determined, it is be set throughout the network operational time. Therefore, the use of this algorithm make it possible to determine the percentage of nodes that will be CHs (PFL), prior to network deployment, without the need to have a specialized equipped node with GPS and without need to transmit node location to the base station. Sampath Priyankara in at al [2] proposed Heterogeneous networks lower the hardware cost and reduce the communication cost of the sensing nodes. Homogeneous networks achieve uniform energy drainage. However both features cannot be incorporated in the same network. The objective of this paper is to design a network architecture which able to maintain above three characteristics in heterogeneous networks. Honggang Zhao in at al [3] in this paper, a more energy-efficient clustering algorithm (EECA) is proposed. The algorithm adopts "seven segments algorithm" and "weighted theory" to predict the packets stream in next operation period, then adjusts operation period adaptively, and create a new cluster-heads selection method to avoid unnecessary information exchanging. Jiuqiang Hana in at al [4] in this paper, the focus was on the unbalanced energy consumption of large scale WSN in environmental monitoring. It is caused by the featured transmission pattern: convergecast. An analytical method is proposed to describe the decline of energy consumption on the radial direction.

\section{PROPOSED WORK}

In Distributed Approach, the study has been made on the implementation of spread clustering protocols in wireless sensor networks. The presentation of two popular schemes, HEED and HIDCA protocols, Node clustering has been extensively deliberate for wireless sensor networks and numerous clustering algorithms have been proposed in the literature, such as LEACH, HEED, and HIDCA. The Highest Identifier Clustering Algorithm (HIDCA), modified from [6] [7], is a primitive clustering protocol. Firstly, throughout the node detection stage, each sensor node replaces information to conclude its neighboring nodes. Then, each node evaluates its ID with those from its neighbors. If its own ID has the minimum number, the node will become the $\mathrm{CHs}$ and all supplementary nodes will demand to connect the cluster and hence grow to be cluster members. Following the cluster is formed, the $\mathrm{CHs}$, that is, the node with lowest ID, sends control packets to continue the process of the cluster. Rejection cluster head rotation is measured in this protocol. The CHs maintain portion for the cluster awaiting its battery power is depleted, during which another surrounding of clustering progression will take place and the node with the second lowest ID will be selected as the CHs. The LEACH joins the Medium Access Control and routing functionalities. In LEACH, clusters are produced based on the most advantageous number of $\mathrm{CHs}$, which is intended using the preceding information of consistent node allocation. The $\mathrm{CHs}$ establish a TDMA agenda for each sensor nodes inside its cluster. Comprehensive synchronization is frequently essential, which consumes important quantity of network 
resources. furthermore, the cluster diameter in LEACH is unspecified to be unrestricted, which might result in the generated CMs being situated distant absent from the $\mathrm{CHs}$ and each other In HEED, clusters are generated lacking any postulation about node distribution. The cluster width is partial and unchanging, and a $\mathrm{CHs}$ revolution scheme is working for load balancing. Though HEED can attain a highquality load balance in a little area, the traffic loads in dissimilar areas are still unstable, consequently foremost to unstable energy utilization in the whole network. It should be pointed out that both LEACH and HEED are CHs -centric algorithms, which initial choose $\mathrm{CHs}$ based on a collection policy, such as the node with the major outstanding energy, and then insert every non-CHs node into the cluster of its adjoining $\mathrm{CHs}$ or the $\mathrm{CHs}$ with some predefined possessions, such as the major node quantity. . The Low-Energy Adaptive Cluster Hierarchy (LEACH) combines the MAC (Medium Access Control) and routing functionalities. In LEACH, clusters are generated based on the optimal number of cluster heads, which is calculated using the prior knowledge of uniform node distribution. The cluster head determines a TDMA schedule for each sensor nodes within its cluster. Global synchronization is usually required, which consumes significant amount of network resources. Moreover, the cluster diameter in LEACH is assumed to be unlimited, which may result in the generated cluster members being located far away from the cluster head and each other. In HEED, clusters are generated without any assumption about node distribution. The cluster diameter is limited and fixed, and a cluster head rotation scheme is employed for load balancing. Although HEED can achieve a good load balance in a small area, the traffic loads in different areas are still unbalanced, thus leading to unbalanced energy consumption in the whole network. It should be pointed out that both LEACH and HEED are cluster head-centric algorithms, which first select cluster heads based on a selection policy, such as the node with the largest residual energy, and then adds each noncluster-head node into the cluster of its nearest cluster head or the cluster head with some predefined property, such as the largest node degree.

\section{EXPERIMENTAL SETUP AND RESULT}

LEACH-distributed versus LEACH-distributed [8][9] In this section is exposed a comparison with the results of the simulations of Normal LEACH, Distributed LEACH, and federal leach. The evaluate consequences as explain above are related to the number of rounds done until half of the nodes are dead or when the first node is dead, where the latter case is also called network lifetime. Evaluation parameter: the effectiveness of the proposed methods is demonstrated by numerical simulation. The proposed methods are compared with the conventional methods LEACH [9]. In the simulation, 25 sensor nodes are aimlessly distributed in the square region of the simulation is performed for $\mathrm{N}=100,300$ and 1000. For Energy Model [9] d075 m ,Eelec $50 \mathrm{~nJ} /$ bit, Efusion $5 \mathrm{~nJ} / \mathrm{bit}$, efs $100 \mathrm{pJ} / \mathrm{bit} / \mathrm{m} 2$, عmp $1.3 \mathrm{fJ} / \mathrm{bit} / \mathrm{m} 4$, Initial battery level 0.5 Joule,Energy for data aggregation $5 \mathrm{~nJ} / \mathrm{bit} /$ signal For Packet Model,Data packet size 800 bit,Broadcast packet size200 bit, Packet header siz 200 bit,For Distributed Method, Rinf 20 meters, Ren 55 meters. Half-dead network and First node dead: It can be seen the dissimilarity in the outcomes of both protocols. In the case of a short steady phase, i.e. composed by frames, the solar-aware extension shows a higher number of rounds attain than the innovative LEACH distributed version. However both show a comparable behavior when the base station (BS) is placed at dissimilar distances, getting worse the farther the BS is to the closest node as can be observed in FND(first node dead) HND(HALF DEAD NODE)

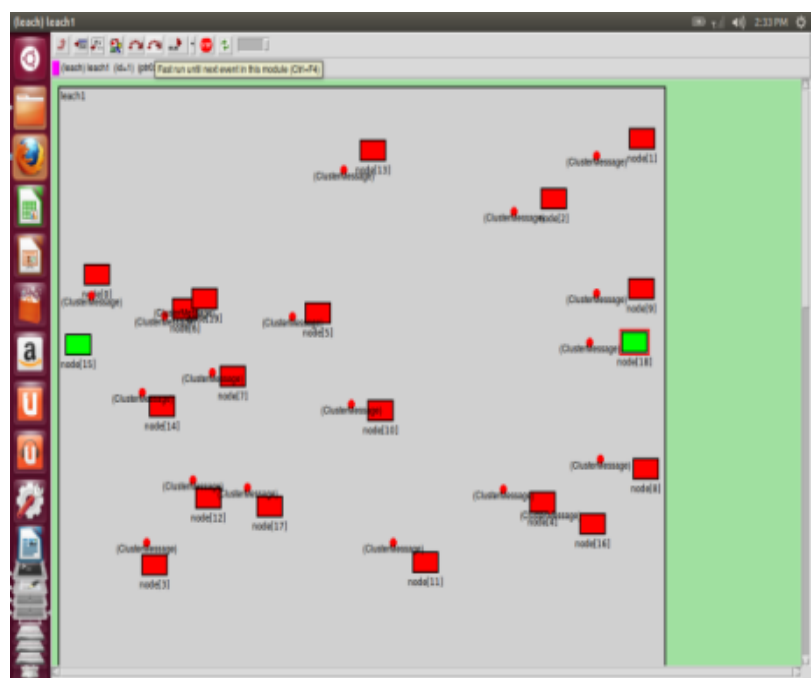

Figure 1. NORMAL LEACH

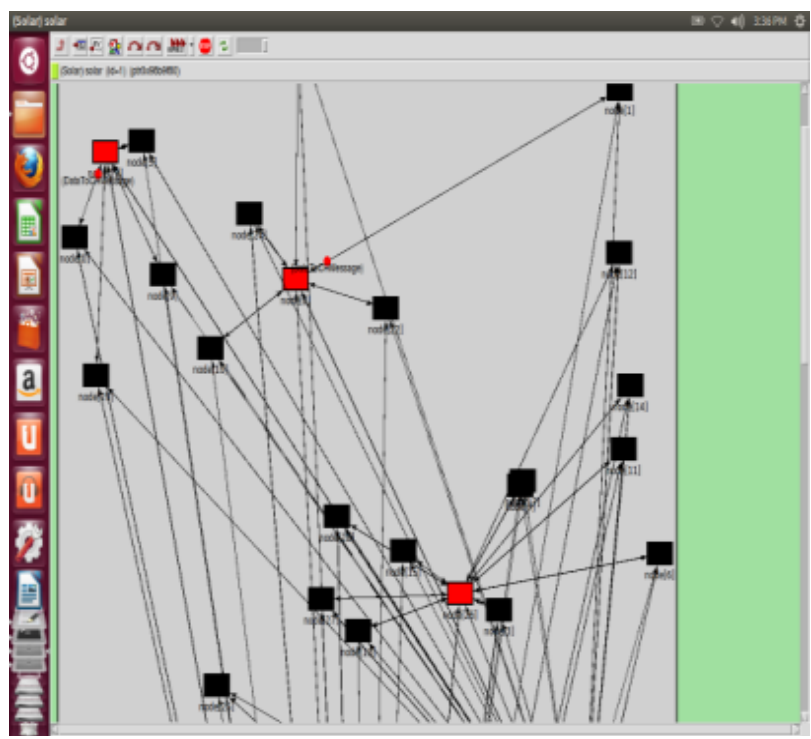

Figure 2. DISTRIBUTED LEACH

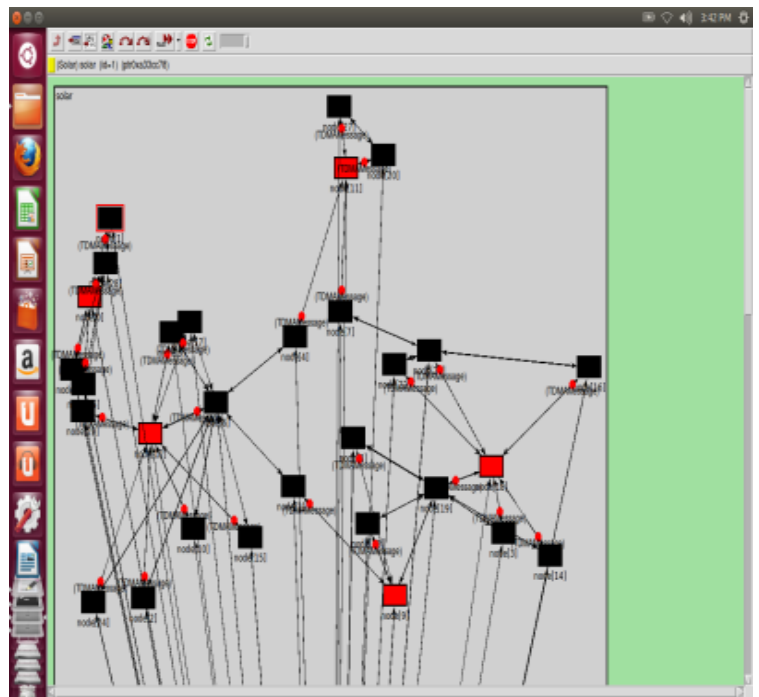

Figure 3. FEDERAL LEACH 
Federal leach comparison Normal Leach:

$\begin{array}{cll}\text { No. of nodes } & \text { FND } & \text { HND } \\ \mathbf{2 0} & \mathbf{2 5} & \mathbf{6 2} \\ \mathbf{4 0} & \mathbf{2 0} & \mathbf{5 8} \\ \mathbf{6 0} & \mathbf{2 5} & \mathbf{5 6} \\ \mathbf{8 0} & \mathbf{2 6} & \mathbf{6 4} \\ \mathbf{1 0 0} & \mathbf{3 0} & \mathbf{7 0}\end{array}$

Federal Leach

No. of nodes FND HND

$20 \quad 79 \quad 112$

$\begin{array}{lll}40 & 78 & 102\end{array}$

$60 \quad 82 \quad 114$

$80 \quad 86 \quad 119$

$\begin{array}{lll}100 & 84 & 111\end{array}$

Improvement of Centralized Leach

$\begin{array}{lll}\text { Nodes } & \text { FND } & \text { HND } \\ 20 & \mathbf{3 . 1 6} & \mathbf{1 . 8 0 6 4 5 1 6 1 2 9} \\ \mathbf{4 0} & \mathbf{3 . 9} & \mathbf{1 . 7 5 8 6 2 0 6 8 9 7} \\ \mathbf{6 0} & \mathbf{3 . 2 8} & \mathbf{2 . 0 3 5 7 1 4 2 8 5 7} \\ \mathbf{8 0} & \mathbf{3 . 3 0 7 6 9 2 3 0 7 7} & \mathbf{1 . 8 5 9 3 7 5} \\ \mathbf{1 0 0} & \mathbf{2 . 8} & \mathbf{1 . 5 8 5 7 1 4 2 8 5 7}\end{array}$

Distributed leach comparison

Normal Leach:

$\begin{array}{cll}\text { No. of nodes } & \text { FND } & \text { HND } \\ \mathbf{2 0} & \mathbf{2 5} & \mathbf{6 2} \\ 40 & \mathbf{2 0} & \mathbf{5 8} \\ \mathbf{6 0} & \mathbf{2 5} & \mathbf{5 6} \\ \mathbf{8 0} & \mathbf{2 6} & \mathbf{6 4} \\ \mathbf{1 0 0} & \mathbf{3 0} & \mathbf{7 0}\end{array}$

Distributed Leach

\begin{tabular}{|cll} 
No. of nodes & FND & HND \\
20 & 112 & 182 \\
40 & 116 & 193 \\
60 & 111 & 181 \\
80 & 120 & 188 \\
100 & 124 & 195
\end{tabular}

Improvement of Distributed Leach

$\begin{array}{lll}\text { Nodes } & \text { FND } & \text { HND } \\ 20 & 4.48 & 2.935483871 \\ 40 & 5.8 & 3.3275862069 \\ 60 & 4.44 & \mathbf{3 . 2 3 2 1 4 2 8 5 7 1} \\ 80 & 4.6153846154 & \mathbf{2 . 9 3 7 5} \\ 100 & 4.1333333333 & \mathbf{2 . 7 8 5 7 1 4 2 8 5 7}\end{array}$

When the distributed leach phase has doubled the number of frames, i.e. the duration of the steady phase is doubled, the behavior of both protocols remain the same but decreasing the number of rounds achieved up to almost the half of them. This circumstances can be explain as an instance of a low-cost set-up phase in energy conditions, but a high-cost steady phase due to a non-optimal election of the cluster heads and the direct communication between cluster heads and base station.

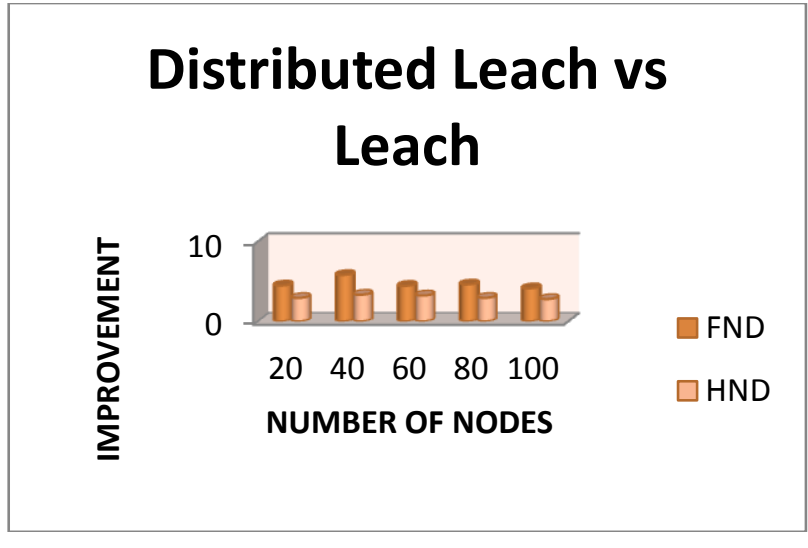

Figure 4. DISTRIBUTED LEACH VS NORMAL LEACH

It can be experiential the consequences of both protocols with the longest distributed phase simulated. The outcome is actually similar to the previous ones as expected, but decreasing the overall amount of rounds achieve.

\section{Federal Leach vs Leach}

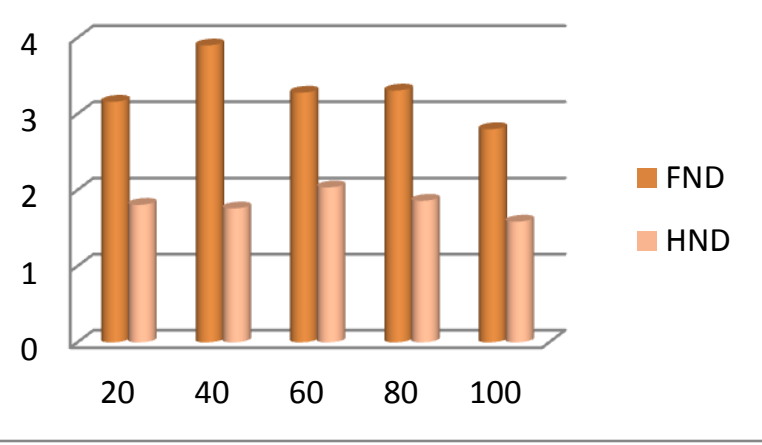

Figure 5. FEDERAL LEACH VS NORMAL LEACH

The consequences of both protocols get closer being still better in the case of Solar-aware LEACH. If the duration of the distributed phase increases, the results of both protocols are really similar as can be observed figure 4 . Even though the Solar-aware LEACH still achieves a longer lifetime, the difference between them is not very noticeable in large area networks, chiefly. Both protocols get worse results the farther is the BS to the closest node.

\section{CONCLUSION}

In this work it has been explain an energy-efficient clustering algorithm in wireless sensor network. Here it is studied classic clustering algorithms in wireless sensor networks and finds two main reasons causing unnecessary energy consumption, which are fixed operation periods and too much information exchanged in cluster-heads selection. Here a clustering method is proposed with less communication overhead for clustering based on federal management in k-means algorithm effective clustering and distributed algorithm. In this work some energy efficient protocols are present which are developed from conventional clustering protocol of Federal and distributed clustering protocol. Simulations are performed on omnet++ and results prove that our proposed protocol is better than Normal LEACH. 


\section{REFERENCES}

[1] Jin-Shyan Lee, Wei-Liang Cheng, "Fuzzy-Logic-Based Clustering Approach for Wireless Sensor Networks Using Energy Predication" IEEE SENSORS JOURNAL, VOL. 12, NO. 9, SEPTEMBER 2012.

[2] Sampath Priyankara, Kazuhiko Kinoshita, Hideki Tode, and Koso Murakami, "A Clustering Method for Wireless Sensor Networks with Heterogeneous Node Types," 18th International Conference on Computer Communications and Networks (IEEE ICCCN 2009), Aug. 2009.

[3] Honggang Zhao, Haoshan Shi, Haoyang Tang," An Energy-Efficient Clustering Algorithm in Wireless Sensor Networks" 978-1-4244-2800-7/09-2009 IEEE.

[4] Jiuqiang Hana, Wei Zhaoa, Mao Zheng," An Analytical Model on Unbalanced Energy Consumption in Large Scale Wireless Sensor Network" Third International Conference on Measuring Technology and Mechatronics Automation- 2011

[5] Kyuhong Lee and Heesang Lee," EECR: Energy Efficient Clustering and Routing Approach for Wireless Sensor Networks" 978-1-4577-1088-9/11 IEEE-2011.
[6] J. Luo and J.-P. Hubaux, "Joint mobility and routing for lifetime elongation in wireless sensor networks," Proceeding of 24th Annual Joint Conference of the IEEE Computer and Communications Societies, 2005, pp. 1735-1746.

[7] S.S. Kulkarni and M. Arumugam, "TDMA service for sensor networks,Proceedings. 24th International Conference on Distributed Computing System Workshops, 2004, pp. 604-609.

[8] Thiemo Voigt, Adam Dunkels, Juan Alonso," Solaraware Clustering inkwireless Sensor Networks" 0-78038623-W04/02004 IEEE.

[9] Noritaka Shigei, Hiromi Miyajima, Hiroki Morishita, Michiharu Maeda," Centralized and Distributed Clustering Methods for Energy Efficient Wireless Sensor Networks" Proceedings of the International MultiConference of Engineers and Computer Scientists 2009 Vol I IMECS 2009, March 18 - 20, 2009, Hong Kong". 